H. Fischer, A. Endmann \& M. Krökel (Hrsg.): Mensch und Computer 2015 - Usability Professionals, Berlin: De Gruyter, S. 84- 94.

\title{
User Experience in Kanban
}

\section{Case Study: Erfahrungen aus dem Relaunch eines Internetportals}

\author{
Jan Uhlenbrok \\ basecom $\mathrm{GmbH}$ \& Co. KG \\ Hannoversche Straße 6-8 \\ 49084 Osnabrück \\ jan@usability3000.de
}

\section{Dominique Winter}

Buhl Data Service GmbH

Am Siebertsweiher 3/5

57290 Neunkirchen

dwinter@buhl-data.com

\author{
Eva-Maria Schön \\ CGI Deutschland Ltd. \& Co. KG \\ Am Sandtorkai 72 \\ 20457 Hamburg \\ eva-maria.schoen@cgi.com
}

\author{
Jörg Thomaschewski \\ Hochschule Emden/Leer \\ Constantiaplatz 4 \\ 26723 Emden \\ joerg.thomaschewski@hs-emden-leer.de
}

\begin{abstract}
Kanban ist eine agile Projektmanagementmethode, die auf geringe Durchlaufzeiten während des Entwicklungsprozesses abzielt. Bereits im Jahre 2013 haben wir uns mit der Herausforderung beschäftigt, wie eine Integration von Human-Centered Design in Kanban gestaltet werden kann. Ein wesentlicher Punkt dabei ist es, einen Gesamtüberblick über die zu bearbeitenden Aufgaben zu erhalten. Hierzu haben wir verschiedene Methoden vorgestellt, welche den Kanban-Prozess um Human-Centered Design Aktivitäten erweitern.

In einer Case Study zum Relaunch eines Internetportals wurden die Methoden im interdisziplinären Team produktiv eingesetzt, um ein Produkt mit positiver User Experience zu entwickeln. Nach Abschluss des Projektes wurden Interviews mit den Projektbeteiligten durchgeführt, Ergebnisse zusammengefasst sowie Vor- und Nachteile herausgearbeitet.
\end{abstract}

\section{Keywords}

Human-Centered Design, User Experience, Kanban, Agile Development, Case Study 


\section{Einleitung}

In der heutigen Zeit setzen zunehmend mehr Unternehmen Kanban für die Entwicklung digitaler Produkte ein. „Kanban und Design Thinking haben in den letzten zweieinhalb Jahren eine deutlich höhere Wachstumsrate an Nutzern erfahren als agile Methoden insgesamt" (Komus et al. 2014). Im direkten Vergleich der Studienergebnisse Status Quo Agile 2012 (Komus 2012) und 2014 (Komus et al. 2014) wird deutlich, dass Kanban zunehmend an Bedeutung gewinnt. Während 2012 noch 24,04\% der Teilnehmer angaben, dass Kanban keine Bedeutung für sie habe, reduzierte sich diese Zahl innerhalb von nur zwei Jahren auf unter 16,52\%. Gleichzeitig stieg der Anteil der Teilnehmer, für die Kanban eine zentrale Bedeutung hat, um 3,57\%.

Die Methode Kanban hat ihren Ursprung in der Automobilbranche bei Toyota. Damit beste Qualität mit niedrigen Kosten und einer möglichst kurzen Durchlaufzeit erzielt werden kann, wird von einer monotonen Arbeitsteilung abgesehen und der Fokus auf den Fluss des Produktes durch den gesamten Produktionsprozess gelegt (Leopold \& Kaltenecker 2012). Die Methode ist von Anderson (Anderson 2011) mit einigen Anpassungen für die IT übernommen worden. IT-Organisationen setzen Kanban für das Projektmanagement im Rahmen der agilen Softwareentwicklung ein. Bei Kanban für die IT liegt der Fokus auf der aktuell zu erledigenden Aufgabe. Dabei wird die Wertschöpfungskette des zu entwickelnden Produktes mit Hilfe eines Kanban-Boards (vgl. Abbildung 1) visualisiert.

\begin{tabular}{|c|c|c|c|c|c|c|}
\hline 4 & & & & & 3 & \\
\hline To do & \multicolumn{2}{|c|}{ Development } & \multicolumn{2}{|c|}{ Test } & \multirow{2}{*}{ Release } & \multirow{2}{*}{ Done } \\
\hline & In Progress & Done & In Progress & Done & & \\
\hline & & & & & & \\
\hline & & & & & & \\
\hline & & & & & & \\
\hline & & & & & & \\
\hline & & & & & & \\
\hline
\end{tabular}

Abbildung 1: Beispiel Kanban-Board

Kanban zeichnet sich durch drei wichtige Bestandteile aus, hierzu zählen: Visualisierung des Workflows, Limit für Work in Progress (WIP) und Lead Time bzw. Cycle Time (Kniberg \& Skarin 2010). Die Visualisierung des Workflows erfolgt in Kanban über ein Kanban-Board. Die einzelnen Spalten zeigen dabei die Aktivitäten der Wertschöpfungskette, welche eine Aufgabe durchlaufen muss, bevor sie erledigt ist. WIP beschreibt die Anzahl an Aufgaben, die zeitgleich mit den vorhandenen Mitarbeitern bearbeitet werden können. Jede Spalte hat 
ihr eigenes WIP-Limit (siehe in Abbildung 1, die beispielhaften Zahlen in der obersten Zeile). Wenn das WIP-Limit überschritten wird, entsteht ein Engpass, der den Aufgabenfluss unterbricht. Die Cycle Time konkretisiert die Zeit, die eine Aufgabe benötigt, bis sie fertig gestellt ist (Kniberg \& Skarin 2010). Hierzu muss die Aufgabe alle Schritte der Wertschöpfungskette durchlaufen haben.

Die im Kanban-Prozess entstehende Fokussierung auf kleinteilige Aufgaben hat den Nachteil, dass die Gesamtbetrachtung des Produktes eingeschränkt wird. Diese Gesamtbetrachtung ist jedoch notwendig für die Entwicklung eines Produktes mit einer positiven User Experience (UX), da beispielsweise nur hierdurch eine sinnvolle Informationsarchitektur erstellt werden kann. Aus diesem Grund stehen Unternehmen vor der Herausforderung Human-Centered Design (HCD) (Deutsches Institut für Normung 2011) in den Entwicklungsprozess mit Kanban zu integrieren. Eine mögliche Lösung hierfür stellt die von Winter et al. (Winter et al. 2013) vorgestellte Kombination verschiedener Methoden zur Erweiterung des Kanban-Prozesses um HCD-Aktivitäten dar.

\section{Integration von UX in Kanban}

Damit der HCD-Prozess in Kanban abgebildet werden kann, erfolgt eine entsprechende Gestaltung der Wertschöpfungskette. Hierzu ist es sinnvoll, konzeptionelle Aufgaben wie beispielsweise Analyse des Nutzungskontexts, Festlegen von Nutzungsanforderungen und Erarbeitung von Gestaltungslösungen zu berücksichtigen. Zudem sollte die Evaluation fest im Prozess verankert werden. Winter et al. (Winter et al. 2013) nehmen für die Umsetzung zum einen Änderungen am Prozess sowie eine Anpassung des Kanban-Boards vor, zum anderen führen sie UX-Artefakte (Persona Stories, Prototypen) ein.

\section{Prozesserweiterung mit Konzeptionsboard}

Ein Kanban-Board bildet in vielen Fällen die Wertschöpfungskette des Software Engineerings ab (vgl. Abbildung 1). Dabei werden konzeptionelle Aufgaben oftmals vernachlässigt, die für eine erfolgreiche Umsetzung der nutzerzentrierten Gestaltung jedoch maßgeblich sind. Damit die konzeptionellen Aufgaben im Kanban-Prozess aufgenommen werden, wird der Prozess nach vorne um ein Konzeptionsboard (vgl. Abbildung 2) erweitert. Das bestehende Kanban-Board der Umsetzung bleibt dabei unberührt. Somit können die konzeptionellen Aufgaben in gleicher Weise wie die Programmieraufgaben organisiert werden. 


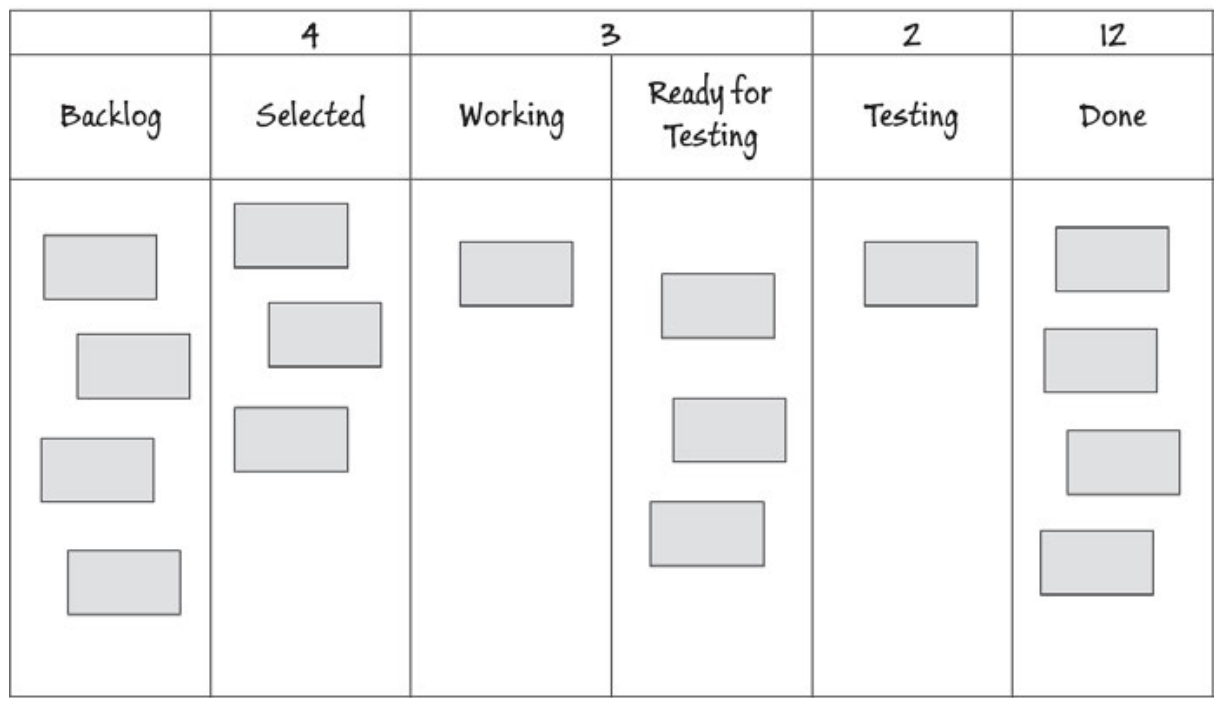

Abbildung 2: Verwendetes Konzeptionsboard

\section{Interdisziplinäre Zusammenarbeit}

Agile Entwicklungsprozesse leben von der Zusammenarbeit verschiedener Disziplinen (Beck et al. 2001). Wenn Experten einer Fachrichtung eng zusammenarbeiten und ein geringer Austausch mit anderen Fachrichtungen stattfindet, können sich funktionale Silos bilden. Diese Bildung kann durch eine interdisziplinäre Zusammenarbeit vermieden werden. Beispielsweise können Konzeptions- und Entwicklungsteams ein gemeinsames Daily Standup ausführen. Hierfür kann das Konzeptionsboard in sichtbarer Nähe zum Entwicklungsboard angebracht werden. Durch den Austausch werden zum einen Engpässe abteilungsübergreifend sichtbar und zum anderen können unterschiedliche Sichtweisen auf ein Problem in den Entwicklungsprozess einfließen.

\section{Release Evaluation}

Ziel einer Release Evaluation ist es, die nutzerbezogenen Produkteigenschaften in regelmäßigen Abständen hinsichtlich ihrer User Experience zu testen. Die resultierenden Optimierungen können als neue Aufgaben in den Kanban-Prozess eingeplant werden. Damit die Release Evaluation in regelmäßigen Abständen stattfindet, wird auch für die letzte Spalte („Done“) des Kanban-Boards ein WIP-Limit eingeführt. Wenn das WIP-Limit erreicht ist, werden entsprechende UX-Tests durchgeführt.

\section{Verwendung von UX-Artefakten}

Damit die am Prozess beteiligten Menschen eine einheitliche Sprache verwenden und eine gemeinsame Vision vom Produkt teilen, ist es wichtig mit den gleichen Artefakten zu 
arbeiten. In agilen Produktentwicklungsprozessen haben sich hierzu Personas, Persona Stories und Prototypen bewährt (Winter et al. 2013).

- Personas repräsentieren den Nutzer während des Entwicklungsprozesses und unterstützen dabei, dass alle Stakeholder ein gemeinsames Verständnis vom Nutzer und seinen Bedürfnissen erhalten (Cooper 1999; Holt et al. 2011; Nielsen 2013;).

- Zur Abgrenzung des Produktumfangs werden in agilen Entwicklungsprozessen häufig User Stories eingesetzt (Cohn 2004; Wirdemann 2011). Persona Stories (Hudson 2013) (auch: Persona-driven User Stories Reichelt 2010; Winter et al. 2012; Holt et al. 2012) sind eine spezielle Form von User Stories, bei denen Personas anstatt von Rollen verwendet werden. Dies hat den Vorteil, dass eine Integration der Personas im gesamten Entwicklungsprozess erfolgt.

- Prototypen visualisieren komplexe Zusammenhänge einzelner Anforderungen (Rudd et al. 1996). Sie ermöglichen es zu lernen, zu entdecken, Ideen zu generieren und zu verfeinern (Lim et al. 2008). Mit ihrer Hilfe kann überprüft werden, ob das konzeptuelle Modell des Produktes mit den Annahmen über das mentale Modell der Nutzer übereinstimmt.

\section{Case Study}

In den Jahren 2013/2014 wurde die zuvor beschriebene Integration von UX in Kanban im Rahmen eines Projektes eingesetzt. Basierend auf (Winter et al. 2013) sind für die Durchführung der Case Study vier Forschungsfragen formuliert worden.

RQ1: Welche Vorteile bringt der Einsatz eines Konzeptionsboards in Kanban?

RQ2: Wie hat sich die interdisziplinäre Zusammenarbeit gestaltet?

RQ3: Wie wurde die Release Evaluation durchgeführt?

RQ4: Wie sind die UX-Artefakte integriert worden?

\section{Projektbeschreibung}

Das Projekt wurde mit einem zwölfköpfigen Team (Teamleiter, Projektmanager, zwei Grafikern, zwei UX-Experten und sechs Entwickler) als Teilprojekt im Rahmen eines Relaunch eines Internetportals innerhalb von sechs Monaten in 2013/2014 durchgeführt. Im Rahmen des Projektes wurde in einer IT-Organisation, die bereits mit Kanban arbeitete, ein Konzeptionsboard eingeführt. Dieses wurde dem bestehenden Entwicklungsboard vorgelagert, so wie bei Winter et al. (Winter et al. 2013) beschrieben.

Aufgrund der Größe des Projektteams wurden damit die nötigen Strukturen für den teamübergreifenden Austausch geschaffen, um die Bildung von funktionalen Silos zu verhindern. 
Für die letzte Spalte des Konzeptionsboards wurde ein WIP-Limit eingeführt. Sobald dieses erreicht wurde, ist eine Release Evaluation durchgeführt worden. Bereits nach einigen Wochen hatte sich die letzte Spalte des Entwicklungsboards gefüllt, so dass eine erste Release Evaluation durchgeführt wurde. Die Schwere der dabei erkannten UX-Probleme wurde von einem UX-Experten bewertet. Schnell umsetzbare Anpassungen $(<30$ Minuten) wurden gemeinsam mit dem Entwickler sofort vorgenommen. Andere Aufgaben wurden dafür unterbrochen. Dieses Vorgehen wurde eingesetzt um Release-Zusagen einzuhalten. War das UX-Problem schwerwiegend, wurde im Konzeptionsboard eine neue Aufgabe erstellt, um das Problem vollumfänglich zu lösen.

Auf die Verwendung von Persona Stories wurde verzichtet, da sich die Nutzung von Personas zum Projektzeitpunkt noch nicht etabliert hatte. Es wurden stattdessen einfache User-Stories eingesetzt. Für komplexere Anforderungen wurden entsprechende Prototypen erstellt, die in der Spalte „Testing“ des Konzeptionsboards (vgl. Abbildung 2) ihre Anwendung fanden.

\section{Datenerhebung und Analyse}

Für die Erhebung aussagekräftiger Daten wurden mit sechs Projektteilnehmern qualitative Telefon-Interviews durch einen neutralen Interviewer (unternehmensextern) durchgeführt. Diese dauerten zwischen 20 und 25 Minuten. In den Interviews wurden die Befragten gebeten, ihre eigene Sicht auf den Prozess zu äußern und eine Einschätzung bezüglich Vorund Nachteilen dieses Vorgehens zu nennen. Abschließend sollten die Befragten ihre Einschätzung der Usability und User Experience des Projektergebnisses äußern und beantworten in welcher Form sich dieses mit durch das sonst übliche Vorgehen erzeugten Projektergebnissen unterschied. Die Telefoninterviews wurden 12 Monate nach Projektabschluss durchgeführt. Das aus dem Projekt entstandene Internetportal hatte sich somit vor der Durchführung der Interviews etabliert.

\section{Ergebnisse}

Die Erkenntnisse aus der Case Study liefern wichtige Hinweise zum Einsatz in der Praxis. Im Folgenden werden die Ergebnisse im Hinblick auf die Forschungsfragen diskutiert. Die Aussagen aus den einzelnen Interviews werden hierzu anonymisiert dargestellt (I1-I6).

\section{Welche Vorteile bringt der Einsatz eines Konzeptionsboards in Kanban?}

- Vorteile vorgelagerte Konzeption: erste Anlaufstelle für Anforderungen, gleiche Anforderungen können gleiche Lösung verwenden (I1)

- Zuvor sind Anforderungen eher unstrukturiert in das Produkt eingeflossen (I1) 
- Zuvor viele Konzepter, die gleichzeitig Anforderungen gestellt haben, jetzt bündeln Konzepter Anforderungen und es erfolgt eine bessere Kommunikation (I5)

- Produktverantwortliche und Konzepter haben Entwicklern viel Chaos erspart (I6)

- Konzeptionsboard für Entwickler nicht sichtbar (I2)

- Konzeptionsarbeit war während des Projektes nicht sichtbar, erst das Ergebnis (I5)

\section{Wie hat sich die interdisziplinäre Zusammenarbeit gestaltet?}

- Hohe Umsetzungsgeschwindigkeit: enge Zusammenarbeit zwischen Entwickler und Konzepter war notwendig, sonst hätte das Projekt 4-5 Monate länger gedauert (I3)

- Zuvor fehlende Verzahnung (räumliche Trennung) führte zu mehr Rückfragen (I3)

- Konzeptionsarbeit war während des Projektes nicht sichtbar, erst das Ergebnis (I5)

- Entwickler bekommen ein besseres Gefühl für die Anforderungen (I2)

- Herausforderung beim Daily Standup: Waage zwischen oberflächliche Betrachtung und detaillierte Diskussion halten (I3)

\begin{tabular}{|l|l|}
\hline Gemeinsame Daily Standup positiv & Gemeinsame Daily Standup negativ: \\
\hline $\begin{array}{l}\text { Transparenz bzgl. der Arbeit anderer (I1), } \\
\text { (I2), (I5) }\end{array}$ & $\begin{array}{l}\text { Uhrzeit und Regelmäßigkeit waren eine } \\
\text { Umstellung (I1) }\end{array}$ \\
\hline $\begin{array}{l}\text { Eigene Arbeit konnte daraufhin besser } \\
\text { abgestimmt werden (I1) }\end{array}$ & $\begin{array}{l}\text { Durch Größe des Teams oftmals 15 min. } \\
\text { überzogen (I1), (I6) }\end{array}$ \\
\hline $\begin{array}{l}\text { Guter Gesamtüberblick: Jeder bekommt } \\
\text { Gefühl, wo Projekt gerade steht (I1) }\end{array}$ & $\begin{array}{l}\text { Es ist blöd, wenn man aus der eigenen } \\
\text { Arbeit gerissen wird (I4) }\end{array}$ \\
\hline $\begin{array}{l}\text { Fördert Kommunikation und Übersicht } \\
\text { (I4) }\end{array}$ & \\
\hline $\begin{array}{l}\text { Zum Teil die Arbeit von Konzeptern } \\
\text { mitbekommen (I4) }\end{array}$ & \\
\hline $\begin{array}{l}\text { Persönliches Stresslevel war geringer, da } \\
\text { die Kommunikation verbessert wurde (I4) }\end{array}$ & \\
\hline Kurze Wege zu Konzeptern (I4) & $\begin{array}{l}\text { Nachfrage in Gruppe während kritischer } \\
\text { Phasen oder Neuerungen (I5) }\end{array}$ \\
\hline $\begin{array}{l}\text { Vorerfahrungen anderer bzgl. komplexer } \\
\text { Aufgaben konnte im Team geteilt werden } \\
\text { (I2) }\end{array}$ & \\
\hline $\begin{array}{l}\text { Für Entwickler interessant zu sehen, wo } \\
\text { die Reise hingeht (aus Usability-Sicht) (I2) }\end{array}$ & \\
\hline
\end{tabular}




\section{Wie wurde die Release Evaluation durchgeführt?}

- Einschätzung der Usability aus Sicht der Projektteilnehmer gut, jeder ist mit Ergebnis zufrieden (I1-I6)

- Zuwachs bzgl. Unique User und Nutzungszahlen (I6)

- Für Zeit und Aufwand ist Ergebnis der Usability sehr gut (I4)

\section{Wie sind die UX-Artefakte integriert worden?}

- Hohe Anzahl an Konzepten ist sehr gut im Ergebnis (I3)

- User Stories kamen aus der Konzeption (I5)

\section{Weitere Erkenntnisse}

- Hohe Effizienz (I1)

- Fokus auf die eigene Arbeit (I1)

- gut zu wissen, dass ein Prozess dahinter steckt, mit Vor- und Nachfolger zur eigenen Position (I1)

- Kanban sehr positiv, da klares Ablaufschema vorhanden (I4)

- Kanban im Vergleich zu Scrum performanter (weniger Organisatorisches) (I3)

- Sehr organisiert im Vergleich zu anderen Projekten ohne agiles Vorgehen (I2)

- Usability: Bisher nicht mit so hoher Genauigkeit und Geschwindigkeit umgesetzt (I3)

- Struktur und Disziplin werden benötigt (I4)

- Im Anschluss an das Projekt können Informationen verloren gehen (I5)

- Mit üblichen Prozess hätte Projekt länger gedauert, Fülle an Funktionen wäre nicht lieferbar (I5)

- Wohl gefühlt, da Anzahl an Aufgaben gut strukturiert (I5)

- Hohe Wertschätzung der eigenen Person (I6)

- Planning Poker hat Spaß gemacht und das Ergebnis des Mittelwertes war gut (I2)

Aus einer Retrospektive mit dem UX-Verantwortlichen gehen weitere Erkenntnisse hervor. Insgesamt konnte bei den Stakeholdern durch die Integration von UX-Experten in den Entwicklungsprozess ein besseres Verständnis für den HCD-Prozess herbeigeführt werden. Zudem wurde das Bewusstsein für die Wichtigkeit eines eigenen, kleinen UX-Teams gestärkt. Weiterhin konnte durch die direkte Zusammenarbeit die Entstehung von funktionalen Silos vermieden werden. Darüber hinaus schärfte die interdisziplinäre 
Zusammenarbeit das Bewusstsein für die Usability bei allen Beteiligten nachhaltig. In aktuellen Projekten äußert sich dies, indem eine gute Bedienbarkeit viel häufiger Gesprächsthema bei den Entwicklern ist. Die Entwickler gehen mit Detailfragen aktiv auf die UX-Experten und Grafiker zu, um eine gemeinsame Lösung zu finden.

Ein weiteres Ergebnis stellt ein bestehender Werkzeugkasten an UI-Elementen dar. Die einzelnen UI-Elemente wurden bezüglich ihrer Eignung im Projekt vom UX-Experten überprüft. Durch diesen Werkzeugkasten an UI-Elementen konnte das Konzeptionsteam nachhaltig entlastet und die Konsistenz der Interaktionselemente vereinheitlicht werden. Die UI-Elemente des Werkzeugkastens werden im aktuell laufenden Betrieb bei kleineren Änderungen vom Entwickler-Team teilweise selbstständig genutzt. Hieraus ergeben sich drei Vorteile: (a) durch die selbständige Verwendung erhalten die Entwickler die Sicherheit auch unter Zeitstress noch immer möglichst passende UI-Elemente zu verwenden, (b) das Konzeptionsteam wird entlastet und (c) die Entwickler haben aufgrund des besseren Verständnisses für den HCD-Prozess und kennen den UX-Experten, so wie der UX-Experte das Projekt kennt.

Bis zum jetzigen Zeitpunkt weist das Internetportal eine vergleichsweise hohe UX und Usability auf, was durch Aussagen von Nutzern und Redakteuren des Internetportals bestätigt wird. Die Anzahl von Nutzerfragen (z.B. „Finde ich ...?“ oder „Wie funktioniert ...?“) ist zurückgegangen, was eine Entlastung des Supports zur Folge hat.

\section{Zusammenfassung und Ausblick}

In der durchgeführten Case Study zum Relaunch eines Internetportals wurden die Methoden zur Integration von HCD in Kanban eingesetzt. Zusammenfassend kann festgehalten werden, dass die Anforderungen durch das vorgelagerte Konzeptionsboard strukturierter in den Entwicklungsprozess eingeflossen sind. Zudem können die konzeptionellen Aufgaben in gleicher Weise wie die Programmieraufgaben organisiert werden. Das gemeinsame Daily Standup sorgt für eine bessere Transparenz bzgl. des Fortschritts und verkürzt die Abstimmungsprozesse zwischen Konzeptern und Entwicklern. Insgesamt bestätigten alle interviewten Projektteilnehmer, dass das Internetportal ein sehr gutes Ergebnis hinsichtlich der wahrgenommenen Usability aufweist. Die Anzahl der erwarteten UX-Probleme hat sich nach subjektiven Empfinden der Stakeholder verringert. Dies lässt sich auf den Einsatz der Prototypen und deren regelmäßiger Verbesserung zurückzuführen. Der entstandene UIWerkzeugkasten wird bis zum jetzigen Zeitpunkt eingesetzt.

Auf Basis der Erkenntnisse lassen sich Empfehlungen für die Optimierung des Vorgehens formulieren. Das Konzeptionsboard war für einige Entwickler nicht sichtbar, da es aufgrund organisatorischer Rahmenbedingungen nicht direkt neben dem Entwicklungsboard platziert werden konnte. Zukünftig kann die Transparenz hinsichtlich der konzeptionellen Aufgaben gesteigert werden, indem das Konzeptionsboard direkt neben dem Entwicklungsboard platziert wird. Aufgrund der großen Teilnehmerzahl während des Daily Standups war die Einhaltung der gesetzten Timebox (15 min.) oftmals nicht möglich. Damit die Timebox 
zukünftig eingehalten werden kann, ist es sinnvoll einen Verantwortlichen zu benennen (vgl. Scrum Master in Scrum, Sutherland \& Schwaber 2013). Aus den Interviews geht zudem der Wunsch hervor, dass die Frequenz der Daily Standups und die Größe des Teilnehmerkreises verringert werden sollte, um die Effizienz zu steigern (vgl. I6).

\section{Literatur}

Anderson, D. J. (2011). Kanban. Evolutionäres Change Management für IT-Organisationen (1st. ed.). Heidelberg, Neckar: dpunkt.

Beck, K., Beedle, M., van Bennekum, A., Cockburn, A., Cunningham, W., Fowler, M., Grenning, J., Highsmith, J., Hunt, A., Jeffries, R., Kern, J., Marick, B., Martin, R., Mellor, S., Schwaber, K., Sutherland, J., \& Thomas, D. (2001). Manifesto for Agile Software Development (2001).

Cohn, M. (2004). User stories applied. For agile software development. Boston, Mass: AddisonWesley.

Cooper, A. (1999). The inmates are running the asylum. Indianapolis, Ind: Sams.

DIN EN ISO 9241-210 (2011). Ergonomie der Mensch-System-Interaktion - Teil 210: Prozess zur Gestaltung gebrauchstauglicher interaktiver Systeme. Beuth, Berlin.

Holt, E.-M., Winter, D. \& Thomaschewski, J. (2011). Personas als Werkzeug in modernen Softwareprojekten. Die Humanisierung des Anwenders. In Usability Professionals 2011, Stuttgart, $40-44$.

Holt, E.-M., Winter, D. \& Thomaschewski, J. (2012). Von der Idee zum Prototypen. Werkzeuge für die agile Welt. In Usability Professionals 2012. German UPA e.V., Stuttgart.

Hudson, W. (201)3. User stories don't help users. Interactions 20, 6 (2013), 50-53.

Kniberg, H. \& Skarin, M. (2010). Kanban and Scrum. Making the most of both. C4Media, Inc.

Komus, A. (2012). Studie: Status Quo Agile. Verbreitung und Nutzen agiler Methoden from www.status-quo-agile.de/

Komus, A., Kuberg, M., Atinc, C., Franner, L., Friedrich, F., Lang, T., Makarova, A., Reimer, D. \& Pabst, J. (2014). Status Quo Agile 2014. Zweite Studie zu Verbreitung und Nutzen agiler Methoden.

Leopold, K. \& Kaltenecker, S. (2012). Kanban in der IT. Eine Kultur der kontinuierlichen Verbesserung schaffen. München: Hanser.

Lim, Y.-K., Stolterman, E. \& Tenenberg, J. (2008). The anatomy of prototypes. Prototypes as Filters, Prototypes as Manifestations of Design Ideas. ACM Trans. Comput.-Hum. Interact. 15, 2 (2008), $1-27$.

Nielsen, L. (2013). Personas from www.interaction-design.org/encyclopedia/personas.html

Reichelt, L. (2010). Persona driven user stories for Agile UX. from www.disambiguity.com/personadriven-user-stories-for-agile-ux/

Rudd, J., Stern, K. \& Isensee, S. (1996). Low vs. high-fidelity prototyping debate. Interactions 3, 1 (1996), 76-85.

Sutherland, J. \& Schwaber, K. (2013). The Scrum Guide. The Definitive Guide to Scrum : The Rules of the Game from www.scrum.org/Portals/0/Documents/Scrum\%20Guides/2013/Scrum-Guide.pdf 
Winter, D., Holt, E.-M. \& Thomaschewski, J. (2012). Persona driven agile development. Build up a vision with personas, sketches and persona driven user stories. Proceedings of the 7th Conference on Information Systems and Technologies (CISTI) (2012).

Winter, D., Schön, E.-M., Uhlenbrok, J. \& Thomaschewski, J. (2013). User Experience in Kanban. Die UX-Karte ausspielen. In Usability Professionals 2013. German UPA e.V., Stuttgart, 220-224.

Wirdemann, R. (2011). Scrum mit User Stories (2nd. ed.). München: Hanser, Carl. 